\title{
Semilinear evolution equations of the parabolic type
}

\author{
by JAN BOCHENEK (Kraków)
}

\begin{abstract}
This paper is devoted to the investigation of the abstract semilinear initial value problem $d u / d t+A(t) u=f(t, u), u(0)=u_{0}$, in the "parabolic" case.
\end{abstract}

1. Introduction. Let $X$ be a Banach space and for each $t \in[0, T]$ let $A(t): X \rightarrow X$ be a closed densely defined linear operator. Let $u$ be an unknown function from $\mathbb{R}$ into $X, f$ be a nonlinear mapping from $\mathbb{R} \times X$ into $X$ and $u_{0} \in X$.

As in [2], we consider the semilinear initial value problem

$$
\left\{\begin{array}{l}
d u / d t+A(t) u=f(t, u), \quad t \in(0, T] \\
u(0)=u_{0} .
\end{array}\right.
$$

Our purpose is to study the existence and uniqueness of solution of (1). Our approach and results look like those in [2], where we discuss the problem in the "hyperbolic" case. The results of this paper generalize some earlier results (cf. for example [3], [4], [6], [7]).

2. Preliminaries. Let $\{A(t)\}, t \in[0, T]$, be a family of operators as in Section 1. We make the following assumptions (cf. [6]).

$\left(\mathrm{Z}_{1}\right) \quad$ The domain $D(A(t))=D$ of $A(t), 0 \leq t \leq T$, is dense in $X$ and independent of $t$.

$\left(\mathrm{Z}_{2}\right) \quad$ For $t \in[0, T]$, the resolvent $R(\lambda: A(t)):=(\lambda-A(t))^{-1}$ of $A(t)$ exists for all $\lambda$ with $\operatorname{Re} \lambda \leq 0$ and there is a constant $M$ such that

$$
\|R(\lambda: A(t))\| \leq \frac{M}{1+|\lambda|} \quad \text { for } \operatorname{Re} \lambda \leq 0, t \in[0, T] .
$$

$\left(\mathrm{Z}_{3}\right) \quad$ There exist constants $K>0$ and $0<\alpha \leq 1$ such that

$$
\left\|(A(t)-A(s)) A^{-1}(\tau)\right\| \leq K|t-s|^{\alpha} \quad \text { for } s, t, \tau \in[0, T] .
$$

1991 Mathematics Subject Classification: 34K30, 34G20, 47D06.

Key words and phrases: semilinear initial value problem, analytic semigroup, classical solution, mild solution. 
REMARK 1 . We note that the assumption $\left(\mathrm{Z}_{2}\right)$ and the fact that $D$ is dense in $X$ imply that for every $t \in[0, T], A(t)$ is the infinitesimal generator of an analytic semigroup $S_{t}(s), s \geq 0$, satisfying

$$
\begin{array}{rlrl}
\left\|S_{t}(s)\right\| & \leq C & \text { for } s \geq 0, \\
\left\|A(t) S_{t}(s)\right\| \leq C / s & \text { for } s>0
\end{array}
$$

here, and in the sequel, we denote by $C$ a generic constant.

TheOREM 1 ([6; Theorem 5.6.1). Under the assumptions $\left(\mathrm{Z}_{1}\right)-\left(\mathrm{Z}_{3}\right)$ there is a unique fundamental solution (evolution system) of $(1), U(t, s)$, on $0 \leq$ $s \leq t \leq T$, satisfying:

(i) $\|U(t, s)\| \leq C$ for $0 \leq s \leq t \leq T$.

(ii) For $0 \leq s \leq t \leq T, U(t, s): X \rightarrow D$ and $t \mapsto U(t, s)$ is strongly differentiable in $X$. The derivative $(\partial / \partial t) U(t, s) \in B(X)$ and it is strongly continuous on $0 \leq s<t \leq T$. Moreover

$$
\begin{gathered}
U(t, t)=I, \quad U(t, s)=U(t, r) U(r, s) \quad \text { for } 0 \leq s \leq r \leq t \leq T \\
\frac{\partial}{\partial t} U(t, s)=-A(t) U(t, s) \quad \text { for } 0 \leq s<t \leq T \\
\left\|\frac{\partial}{\partial t} U(t, s)\right\|=\|A(t) U(t, s)\| \leq \frac{C}{t-s}
\end{gathered}
$$

and

$$
\left\|A(t) U(t, s) A^{-1}(s)\right\| \leq C \quad \text { for } 0 \leq s \leq t \leq T .
$$

(iii) For every $v \in D$ and $t \in(0, T], U(t, s) v$ is differentiable with respect to $s$ on $0 \leq s \leq t \leq T$ and

$$
\frac{\partial}{\partial s} U(t, s) v=U(t, s) A(s) v .
$$

Lemma 1. Under assumptions $\left(\mathrm{Z}_{1}\right)-\left(\mathrm{Z}_{3}\right)$ for all $x \in X$ and $0 \leq s<t \leq T$, $\int_{t_{0}}^{t} U(t, s) x d s \in D$ and there is a constant $M$ such that

$$
\left\|A(t) \int_{t_{0}}^{t} U(t, s) x d s\right\| \leq M\left(t-t_{0}\right)^{\alpha}\|x\|+\left\|\left[I-S_{t}\left(t-t_{0}\right)\right] x\right\|,
$$

where $\alpha \in(0,1]$ is from $(3)$.

Proof. For every $x \in X$ and each $t \in(0, T]$ by (ii) and closedness of the operator $A(t), t \in[0, T]$, for each $\varepsilon \in\left(0, t-t_{0}\right)$ we get

$$
\int_{t_{0}}^{t-\varepsilon} U(t, s) x d s \in D
$$


and

$$
A(t) \int_{t_{0}}^{t-\varepsilon} U(t, s) x d s=\int_{t_{0}}^{t-\varepsilon} A(t) U(t, s) x d s=\int_{t_{0}}^{t-\varepsilon}-\frac{\partial U}{\partial t}(t, s) x d s .
$$

By construction of the fundamental solution $U(t, s)$ we have

$$
U(t, s)=S_{s}(t-s)+W(t, s),
$$

where $S_{t}(s)$ is the analytic semigroup generated by $A(t)$ and $W(t, s)$ is strongly continuously differentiable in $t$ for $0 \leq s<t \leq T$ and

$$
\left\|\frac{\partial W}{\partial t}(t, s)\right\| \leq C(t-s)^{\alpha-1} .
$$

Therefore

$$
\lim _{\varepsilon \rightarrow 0} \int_{t_{0}}^{t_{0}-\varepsilon} \frac{\partial W}{\partial t}(t, s) x d s=\int_{t_{0}}^{t} \frac{\partial W}{\partial t}(t, s) x d s, \quad x \in X
$$

(cf. [6; Sec. 5.6 and 5.7]). Next

$$
\begin{aligned}
\int_{t_{0}}^{t-\varepsilon} \frac{\partial}{\partial t} S_{s}(t, s) x d s= & \int_{t_{0}}^{t-\varepsilon}-A(s) S_{s}(t-s) x d s \\
= & \int_{t_{0}}^{t-\varepsilon}-A(t) S_{s}(t-s) x d s \\
& +\int_{t_{0}}^{t-\varepsilon}\left[A(t) S_{t}(t-s)-A(s) S_{s}(t-s)\right] x d s
\end{aligned}
$$

Since by [6; p. 169],

$$
\left\|A(t) S_{t}(t-s)-A(s) S_{s}(t-s)\right\| \leq C(t-s)^{\alpha-1}
$$

and

$$
\int_{t_{0}}^{t-\varepsilon} A(t) S_{t}(t-s) x d s=\int_{t_{0}}^{t-\varepsilon} \frac{\partial}{\partial s} S_{t}(t-s) x d s=\left[S_{t}(\varepsilon)-S_{t}\left(t-t_{0}\right)\right] x
$$

we get

$$
\text { (18) } \begin{aligned}
\lim _{\varepsilon \rightarrow 0} \int_{t_{0}}^{t-\varepsilon} \frac{\partial U}{\partial t}(t, s) x d s= & \int_{t_{0}}^{t}\left[A(t) S_{t}(t-s)-A(s) S_{s}(t-s)\right] x d s \\
& +\int_{t_{0}}^{t} \frac{\partial W}{\partial t}(t, s) x d s+\left[I-S_{t}\left(t-t_{0}\right)\right] x, \quad x \in X .
\end{aligned}
$$


Returning to (13) we can now conclude that

$$
A(t) \int_{t_{0}}^{t-\varepsilon} U(t-s) x d s
$$

converges as $\varepsilon \rightarrow 0$ and since

$$
\int_{t_{0}}^{t-\varepsilon} U(t-s) x d s \rightarrow \int_{t_{0}}^{t} U(t-s) x d s
$$

as $\varepsilon \rightarrow 0$, it follows from the closedness of $A(t)$ that

$$
\int_{t_{0}}^{t} U(t-s) x d s \in D
$$

and

$$
\begin{aligned}
A(t) \int_{t_{0}}^{t} U(t-s) x d s= & \int_{t_{0}}^{t}\left[A(t) S_{t}(t-s)-A(s) S_{s}(t-s)\right] x d s \\
& -\int_{t_{0}}^{t} \frac{\partial W}{\partial t}(t, s) x d s-\left[I-S_{t}\left(t-t_{0}\right)\right] x .
\end{aligned}
$$

Now (19) together with (15) and (16) yields (11) with $M=2 C / \alpha$ and the proof is complete.

Lemma 2 ([5; Lemma 3]). Let $h: \Delta_{T}=\left\{(t, s) \in \mathbb{R}^{2}: 0 \leq s \leq t \leq T\right\} \rightarrow X$ and suppose that:

(i) for almost all $s \in[0, t]$ the function $[0, T] \ni t \mapsto h(t, s)$ is continuous,

(ii) for each $t \in[0, T], h(t, \cdot)$ is summable over $[0, t]$,

(iii) there exists $N>0$ such that $\|h(t, s)\| \leq N$ for $(t, s) \in \Delta_{T}$.

Then the function $G:[0, T] \rightarrow X$ defined as

$$
G(t)=\int_{t_{0}}^{t} h(t, s) d s, \quad t_{0} \geq 0,
$$

is continuous.

Lemma 3. Suppose that:

(i) assumptions $\left(\mathrm{Z}_{1}\right)-\left(\mathrm{Z}_{3}\right)$ hold,

(ii) a function $g:[0, T] \rightarrow X$ satisfies the Hölder condition with $L>0$ and $0<\theta \leq 1$.

Then for every fixed $t_{0} \in[0, T)$ the formula

$$
v(t)=\int_{t_{0}}^{t} U(t, s) g(s) d s, \quad t \in\left[t_{0}, T\right],
$$


defines a function from $\left[t_{0}, T\right]$ into $D$, the function $w:\left[t_{0}, T\right] \rightarrow X$ given by

$$
w(t)=A(t) \int_{t_{0}}^{t} U(t-s) g(s) d s=A(t) v(t)
$$

is continuous and

(23) $\left\|A(t) \int_{t_{0}}^{t} U(t, s) g(s) d s\right\| \leq\left(M+\frac{C}{\theta} L\right)\left(t-t_{0}\right)^{\beta}+\left\|\left[I-S_{t}\left(t-t_{0}\right)\right] g(t)\right\|$,

where $M>0$ does not depend on $t$, and $\beta=\min (\alpha, \theta)$.

Proof. Let $\varepsilon \in\left(0, t-t_{0}\right)$ be arbitrary. Setting

$$
v_{\varepsilon}(t)=\int_{t_{0}}^{t-\varepsilon} U(t, s) g(s) d s
$$

we obtain

$$
v_{\varepsilon}(t)=\int_{t_{0}}^{t-\varepsilon} U(t, s)[g(s)-g(t)] d s+\int_{t_{0}}^{t-\varepsilon} U(t, s) g(t) d s .
$$

The Hölder continuity of $g$ and the estimate $\|A(t) U(t, s)\| \leq C(t-s)^{-1}$ imply that the function $A(t) U(t, s)[g(s)-g(t)]$ is integrable in $s$ over $\left[t_{0}, t\right]$ and therefore

$$
\lim _{\varepsilon \rightarrow 0} \int_{t_{0}}^{t-\varepsilon} A(t) U(t, s)[g(s)-g(t)] d s=\int_{t_{0}}^{t} A(t) U(t, s)[g(s)-g(t)] d s .
$$

It follows by closedness of $A(t)$ that

$$
\int_{t_{0}}^{t} U(t, s)[g(s)-g(t)] d s \in D
$$

and

(27) $\left\|A(t) \int_{t_{0}}^{t} U(t, s)[g(s)-g(t)] d s\right\| \leq C L \int_{t_{0}}^{t}(t-s)^{\theta-1} d s=\frac{C}{\theta} L\left(t-t_{0}\right)^{\theta}$.

By Lemma 1 we have

$$
\lim _{\varepsilon \rightarrow 0} \int_{t_{0}}^{t-\varepsilon} A(t) U(t, s) g(t) d s=\int_{t_{0}}^{t} A(t) U(t, s) g(t) d s .
$$

This implies

$$
\int_{t_{0}}^{t} U(t, s) g(t) d s \in D
$$


and by (11),

$$
\left\|A(t) \int_{t_{0}}^{t} U(t, s) g(t) d s\right\| \leq M\left(t-t_{0}\right)^{\alpha}+\left\|\left[I-S_{t}\left(t-t_{0}\right)\right] g(t)\right\| .
$$

Now (27) and (29) imply (23) with $\beta=\min (\alpha, \theta)$.

To prove the continuity of the function $w:[0, T] \rightarrow X$, we define the family $\left\{w_{\varepsilon}\right\}_{\varepsilon>0}$ of functions such that

$$
w_{\varepsilon}(t):= \begin{cases}A(t) v_{\varepsilon}(t) & \text { for } \varepsilon \leq t \leq T, \\ 0 & \text { for } 0 \leq t<\varepsilon,\end{cases}
$$

where $v_{\varepsilon}$ is defined by (24) with $t_{0}=0$.

Since for fixed $\varepsilon>0$ we have

$$
\|A(t) U(t, s) g(s) d s\| \leq C / \varepsilon \quad \text { for } 0 \leq s \leq t-\varepsilon<t \leq T,
$$

it is easy to see that, by Lemma 2 , the function $w_{\varepsilon}$ is continuous in $[0, T]$.

Now observe that

$$
\left\|w(t)-w_{\varepsilon}(t)\right\|=\left\|A(t) \int_{t-\varepsilon}^{t} U(t, s) g(s) d s\right\| \leq \widetilde{M} \varepsilon^{\beta}+\left\|\left[I-S_{t}(\varepsilon)\right] g(t)\right\| .
$$

This implies that $w_{\varepsilon}$ tends to $w$ as $\varepsilon \searrow 0$, uniformly with respect to $t \in[0, T]$, and so $w$ is continuous in $[0, T]$. Lemma 3 is proved.

Definition 1. A function $u:[0, T] \rightarrow X$ is said to be a classical solution of (1) if $u$ is continuous on $[0, T]$, continuously differentiable on $(0, T], u(t) \in$ $D(A(t))$ for $0<t \leq T$ and (1) is satisfied.

Using Lemma 3 and Theorem 1 of [2], as a corollary we get

Theorem 2 ([6; Theorem 5.7.1]). Let $\{A(t)\}_{t \in[0, T]}$ satisfy assumptions $\left(\mathrm{Z}_{1}\right)-\left(\mathrm{Z}_{3}\right)$ and let $U(t, s)$ be a fundamental solution of (1). If $g$ is Hölder continuous on $[s, T], s \in[0, T)$, then the initial value problem

$$
\left\{\begin{array}{l}
d u / d t+A(t) u=g(t) \quad \text { for } 0 \leq s<t \leq T, \\
u(s)=x
\end{array}\right.
$$

has, for every $x \in X$, a unique classical solution u given by

$$
u(t)=U(t, s) x+\int_{s}^{t} U(t, \tau) g(\tau) d \tau .
$$

3. The main theorem. In this section we consider the nonlinear problem (1) mentioned in the introduction. Suppose that the family $\{A(t)\}_{t \in[0, T]}$ satisfies assumptions $\left(\mathrm{Z}_{1}\right)-\left(\mathrm{Z}_{3}\right)$ and let $f:[0, T] \times X \rightarrow X$ be continuous.

Similarly to the linear case we have 
Theorem 3. If $f:[0, T] \times X \rightarrow X$ is continuous and $u$ is a solution of the problem (1), then $u$ satisfies the integral equation

$$
u(t)=U(t, 0) u_{0}+\int_{0}^{t} U(t, s) f(s, u(s)) d s,
$$

where $U(t, s)$ is the fundamental solution of (1).

TheOREM 4. Let assumptions $\left(\mathrm{Z}_{1}\right)-\left(\mathrm{Z}_{3}\right)$ hold and let $f:[0, T] \times X \rightarrow X$ be continuous. Suppose that there exists $N>0$ such that

$$
\|f(t, x)-f(t, y)\| \leq N\|x-y\| \quad \text { for } t \in[0, T], x, y \in X .
$$

Then for every $u_{0} \in X$ there exists a unique continuous function $u:[0, T] \rightarrow$ $X$ that solves the integral equation (32).

The proof of this theorem is the same as the proof of Theorem 3 in [2] and is omitted.

Definition 2. A continuous solution of the integral equation (32) is said to be a mild solution of the problem (1) (cf. [6; Def. 6.1.1, p. 184]).

Theorem 5. Assume $\left(\mathrm{Z}_{1}\right)-\left(\mathrm{Z}_{3}\right)$ and let $u_{0} \in D$. Let $f:[0, T] \times X \rightarrow X$ satisfy the condition

$$
\|f(t, x)-f(r, y)\| \leq N\left(|t-r|^{\theta}+\|x-y\|\right) \quad \text { for } t, r \in[0, T], x, y \in X,
$$

where $N>0, \theta \in(0,1]$. Then the problem (1) has a unique classical solution which is a solution of the integral equation (32).

The proof of this theorem is based on the following lemmas (cf. [2, Lemmas 4 and 5]).

Lemma 4. Suppose that:

(i) $f:[0, T] \times X \rightarrow X$ is continuous,

(ii) there exists $N>0$ such that $\|f(t, x)-f(t, y)\| \leq N\|x-y\|$ for $t \in[0, T], x, y \in X$,

(iii) $u_{n}:[0, T] \rightarrow X$ is continuous for each $n \in \mathbb{N}$,

(iv) $\left\{u_{n}\right\}$ is uniformly convergent in $[0, T]$.

Then there exists a constant $C>0$ such that

$$
\left\|f\left(t, u_{n}(t)\right)\right\| \leq C \quad \text { for } t \in[0, T] \text { and } n \in \mathbb{N} .
$$

REMARK 2. Lemma 4 is true without the assumption (ii).

Lemma 5. Under the assumptions of Theorem 5 the solution $u$ of the equation (32) satisfies the Lipschitz condition on $[0, T]$. 
The proof of Lemma 5, using the inequality (23), is almost the same as the proof of Lemma 5 in [2].

REMARK 3 . The assumption " $u_{0} \in D$ " is necessary in Lemma 5 because for $x \in X$, the function $u=U(t, 0) x, t \in[0, T]$, may not be Lipschitz continuous.

Proof of Theorem 5. The assumption $u_{0} \in D$ and Lemma 5 imply that the solution $u$ of the integral equation (32) satisfies the Lipschitz condition. From this by (33) we deduce that the mapping $[0, T] \ni t \mapsto f(t, u(t))$ satisfies the Hölder condition.

Then, by Theorem 2, $u$ is a solution of the equation

$$
d z / d t+A(t) z=f(t, u(t)), \quad t \in(0, T],
$$

with the initial condition $z(0)=u_{0}$. This means that $u$ is a solution of the problem (1), if $u_{0} \in D$.

The uniqueness for the problem (1) follows immediately from the uniqueness of the solution of (32).

EXAMPLE ([7; p. 129]). Let $\Omega$ be a bounded region in $\mathbb{R}^{n}$ with boundary $\partial \Omega$ of class $C^{m}$. Consider the following mixed problem:

$$
\left\{\begin{array}{l}
\frac{\partial u}{\partial t}(t, x)+\mathcal{A}(t, x, D) u(t, x)=f(t, x, u(t, x)), \quad x \in \Omega, 0<t \leq T \\
u(0, x)=u_{0}(x), x \in \Omega \\
\frac{\partial^{\alpha} u}{\partial x^{\alpha}}(t, x)=0, \quad|\alpha| \leq m / 2-1, x \in \partial \Omega, 0<t \leq T
\end{array}\right.
$$

where

$$
\mathcal{A}(t, x, D):=\sum_{|\alpha| \leq m} a_{\alpha}(t, x) D^{\alpha}
$$

is a strongly elliptic differential operator, uniformly in $t \in[0, T]$. We assume that for each $t \in[0, T]$ the coefficients $a_{\alpha}$ for $|\alpha|=m$ are continuous in $\bar{\Omega}$ and the other coefficients are bounded and measurable in $\Omega$. We also assume the coefficients to satisfy Hölder's condition in $t$ with exponent $h \in(0,1)$ uniformly, i.e.

$$
\max _{|\alpha| \leq m} \sup _{x \in \Omega}\left|a_{\alpha}(t, x)-a_{\alpha}(s, x)\right| \leq L|t-s|^{h} .
$$

Set $X=L^{p}(\Omega), D(A(t))=W_{p}^{m}(\Omega) \cap \stackrel{\circ}{W}_{p}^{m / 2}(\Omega)$ with $1<p<\infty$ and $A(t): D(A(t)) \rightarrow X$, where $(A(t) u)(x):=\mathcal{A}(t, x, D) u(x)$ for $t \in[0, T]$, $x \in \Omega$. Then the mixed problem (34) may be considered as an abstract semilinear parabolic problem of the form (1) in the Banach space $X=$ 
$L^{p}(\Omega)$, where $u_{0}=u_{0}(x), x \in \Omega$, and $f:[0, T] \times X \rightarrow X$ is defined by

$$
f(t, u)(x):=f(t, x, u(x)), \quad t \in[0, T], x \in \Omega .
$$

We assume that:

(i) $u_{0}(\cdot) \in W_{p}^{m}(\Omega) \cap \stackrel{\circ}{W}_{p}^{m / 2}(\Omega)=D(A(t))$.

(ii) $f$ satisfies the Hölder condition with exponent $0<\theta \leq 1$ :

$$
\sup _{x \in \Omega}|f(t, x, u(x))-f(s, x, u(x))| \leq M|t-s|^{\theta}, \quad u \in L^{p}(\Omega) .
$$

(iii) $f$ satisfies the Lipschitz condition in $X=L^{p}(\Omega)$ with respect $u \in$ $L^{p}(\Omega)$, i.e.

$$
\|f(t, u)-f(t . v)\|_{L^{p}(\Omega)} \leq L\|u-v\|_{L^{p}(\Omega)} \quad \text { for } t \in[0, T] .
$$

Theorem 5 shows that under the above assumptions the problem (34) has a unique solution $u=u(t, x),(t, x) \in[0, T] \times \Omega$, with $u(\cdot, x) \in C^{1}(0, T) \cap$ $C^{0}[0, T]$ for each $x \in \Omega$ and $u(t, \cdot) \in W_{p}^{m}(\Omega) \cap \stackrel{\circ}{W}_{p}^{m / 2}(\Omega)=D(A(t))$ for each $t \in[0, T]$.

REMARK 4. The results of this paper apply to semilinear parabolic differential equations or systems of equations of arbitrary order. They were obtained basing on the theory of semigroups of bounded linear operators in Banach space.

Of course, in the mathematical literature there are many results on existence and uniqueness of solutions of more general nonlinear parabolic equations and systems. Investigation of those problems requires different techniques. For example in [1] some results are proved on local existence of continuously differentiable solutions of quasilinear parabolic systems under general nonlinear boundary conditions.

These results are different from ours and difficult to compare with them.

\section{References}

[1] P. Acquistapace and B. Terreni, On quasilinear parabolic systems, Math. Ann. 282 (1988), 315-335.

[2] J. Bochenek, An abstract semilinear first order differential equation in the hyperbolic case, Ann. Polon. Math. 61 (1995), 13-23.

[3] D. Henry, Geometric Theory of Semilinear Parabolic Equations, Lecture Notes in Math. 840, Springer, Berlin, 1981.

[4] T. Kato, Perturbation Theory of Linear Operators, Grundlehren Math. Wiss. 132, Springer, New York, 1980.

[5] M. Kozak, An abstract nonlinear temporally inhomogeneous equation, Demonstratio Math. 23 (1990), 993-1003. 
[6] A. Pazy, Semigroups of Linear Operators and Applications to Partial Differential Equations, Springer, 1983.

[7] H. Tanabe, Equations of Evolution, Pitman, London, 1979.

Institute of Mathematics

Cracow University of Technology

Warszawska 24

31-155 Kraków, Poland

Reçu par la Rédaction le 9.10.1997

Révisé le 3.9.1999 